\title{
Neutrophil-to-lymphocyte ratio has limited predictive value for determining outcomes in perforated colon cancer: a retrospective study.
}

\section{Chunyi Wu ( $\square$ chunyiwu237@gmail.com )}

Chang Gung Memorial Hospital Linkou Main Branch: Chang Gung Memorial Hospital https://orcid.org/0000-00019200-0013

Jy-Ming Chiang

Chang Gung Memorial Hospital Linkou Main Branch: Chang Gung Memorial Hospital

\section{Jeng-Fu You}

Chang Gung Memorial Hospital Linkou Main Branch: Chang Gung Memorial Hospital

\section{Reiping Tang}

Chang Gung Memorial Hospital Linkou Main Branch: Chang Gung Memorial Hospital Jinn-Shiun Chen

Chang Gung Memorial Hospital Linkou Main Branch: Chang Gung Memorial Hospital

\section{Chien-Yuh Yeh}

Chang Gung Memorial Hospital Linkou Main Branch: Chang Gung Memorial Hospital

\section{Pao-Shiu Hsieh}

Chang Gung Memorial Hospital Linkou Main Branch: Chang Gung Memorial Hospital

Wen-Sy Tsai

Chang Gung Memorial Hospital Linkou Main Branch: Chang Gung Memorial Hospital

Hsin-Yuan Hung

Chang Gung Memorial Hospital Linkou Main Branch: Chang Gung Memorial Hospital

\section{Sum-Fu Chiang}

Chang Gung Memorial Hospital Linkou Main Branch: Chang Gung Memorial Hospital

Cheng-Chou Lai

Chang Gung Memorial Hospital Linkou Main Branch: Chang Gung Memorial Hospital

\section{Research}

Keywords: neutrophil-to-lymphocyte ratio; perforated colon cancer; leukocytosis; serum albumin; prognostic factor

Posted Date: September 8th, 2020

DOI: https://doi.org/10.21203/rs.3.rs-70836/v1

License: (c) (i) This work is licensed under a Creative Commons Attribution 4.0 International License. Read Full License 


\section{Abstract}

Background: The neutrophil-to-lymphocyte ratio (NLR) is a significant prognostic marker in resectable colorectal cancer; however, there are no equivalent findings for perforated colon cancer. Using our colorectal cancer database, we retrospectively analyzed the data from 1995 to 2015 to determine if preoperative NLR was associated with survival outcomes in patients with perforated colon cancer.

Methods: One-to-one propensity score matching (PSM) was applied to minimize the difference between the higher- $(>5)$ and lower- $(\leq 5)$ NLR groups. Clinicopathological factors, long-term overall survival (OS), and disease-free survival (DFS) were analyzed and compared between the two groups. The main outcome measures were OS and DFS.

Results: Before PSM, the higher-NLR group had significantly higher prevalence of leukocytosis (lower- vs. higher-NLR groups: 12 [12.9\%] vs. 46 [59.7\%], p<0.001), lower serum albumin levels (lower- vs. higher-NLR groups: 30 [32.3\%] vs. 42 [54.5\%], $p=0.003$ ), and higher emergent operation rate (lower- vs. higher-NLR groups: 5 [5.4\%] vs. 20 [26.0\%], $p<0.001$ ). After one-to-one PSM, the groups comprised 41 patients each; none of the parameters were significantly different. The mean follow-up period was 76.3 months. Five-year OS and DFS were not significantly different (OS: $p=0.637$; DFS: $\mathrm{p}=0.827$ ) between the higher- and lower-NLR groups.

Conclusion: NLR have limited predictive value for outcomes in patients with perforated colon cancer.

\section{Background}

Colon cancer is one of the most common causes of cancer-related deaths worldwide, with an estimated 551,269 deaths and 1,096,601 new colon cancer cases diagnosed annually [1]. Although current cancer treatments have improved the outcomes, long-term survival remains unsatisfactory. Increasing reports have shown that systemic inflammatory reactions increase the risk of cancer development [2,3]. A number of studies have focused on inflammation-related prognostic variables, such as C-reactive protein and albumin levels, platelet-to-lymphocyte ratio, and neutrophil-to-lymphocyte ratio (NLR), to identify patients at a high risk of colorectal cancer recurrence after primary surgery [4-7].

The NLR is a significant prognostic marker in patients with resectable colorectal cancer. NLR is considered not only a tumor-related inflammatory environment marker but also an indicator of anti-tumor host immunity. A high pre-operative $\operatorname{NLR}(>5)$ has been associated with poor overall survival [8-10].

Preoperative NLR has been shown to influence disease-free survival in patients with stage I to III colorectal cancer; an elevated NLR (estimated cut-off value > 3 ) is also associated with worse outcomes (5-year disease-free survival: $66.3 \%$ vs. $78.9 \%$ for colon cancer; $60.5 \%$ vs. $66.2 \%$ for rectal cancer) [11].

However, there is a lack of evidence on the association between perforated colon cancer and the NLR. Therefore, we aimed to determine the association between preoperative NLR, clinicopathologic features, and survival outcomes in patients with perforated colon cancer.

\section{Materials And Methods}

The medical records of a total of 240 patients with pathologically confirmed perforated colon cancer who underwent surgical treatment at Chang Gung Memorial Hospital, Linkou, between January 1, 1995 and December 31, 2015, were reviewed. All data were retrospectively reviewed from a prospectively maintained database after obtaining the institutional review board approval. The definition of perforated colon was based on the pathology reports mentioning gross or microscopic perforation. We excluded patients with post-polypectomy perforation, those with short-term mortality $(<30$ days postoperatively), and those who had preoperative distal metastasis. Preoperative evaluation and postoperative 
follow-up data were collected for analysis. Data on the date, tumor stage and location, treatments, and cause of death (recurrence vs. second primary cancer) were also collected.

Preoperative patients' demographic characteristics included age, sex, comorbid diseases (hepatitis, diabetes status, and liver cirrhosis), serum albumin, carcinoembryonic antigen, and hemoglobin levels, white blood cell (WBC) count, and NLR. Perioperative factors included tumor location and whether the patient underwent an emergent operation or not. Tumor TNM staging, histology, and pathological features were also reviewed.

The NLR was calculated by dividing the number of neutrophils by the number of lymphocytes per the serum differential count. Preoperative NLR was calculated using the latest preoperative data, either at the emergency department or during admission. The cut-off value for the NLR in this study was defined as 5.0, which was equal to the cut-off value of the most reviewed articles.

The primary study outcomes were overall survival and disease-free survival. The cause of death was recorded as an event. Patients were recorded alive at the last follow-up, regardless of their disease status. Survival was measured from the operation date to the date of death or last known follow-up.

Propensity scores were calculated using the following covariates: WBC count, albumin level, and emergent surgery. The propensity scores were used for one-to-one patient matching between the higher- and lower-NLR groups; the matching tolerance was defined as 0.05 .

\section{Statistical analysis}

Statistical analyses were performed using SPSS version 24.0 (IBM Corp., Armonk, NY), and p-values $<0.05$ were considered statistically significant. Pearson's chi-squared test was used for clinicopathologic descriptive data to determine the differences between the higher- and lower-NLR groups. Kaplan-Meier survival curves were used to assess survival in the different subgroups. The Cox proportional hazards model was used for univariate and multivariate analyses; variables with $p<0.05$ in the univariate analysis were evaluated further in the multivariate analyses to determine the independent risk factors for survival.

\section{Results}

All 240 patients underwent curative-intended surgical treatments, including laparoscopic colectomy, traditional (open) colectomy, and emergent exploratory laparotomy. Patients were excluded because they underwent polypectomy and surgery within 24 hours $(n=5)$, had early mortality ( $<30$ days postoperatively, $n=4)$, and had confirmed distal metastasis $(n=61)$ (Fig. 1). A total of 170 patients were ultimately enrolled in this study. There were $93(54.7 \%)$ patients with lower NLR and 77 (45.3\%) patients with higher NLR. The mean follow-up time was 73.5 months, ranging from 1.7 to 135.7 months.

Initial clinicopathological factors were compared between the higher- and lower-NLR groups (Table 1). In the higher-NLR group, there was a higher prevalence of leukocytosis (WBC count $>12000 / \mu \mathrm{L}$, lower- vs. higher-NLR groups: 12 [12.9\%] vs. $46[59.7 \%], p<0.001)$, a lower mean serum albumin level (albumin < $3.5 \mathrm{~g} / \mathrm{dL}$, lower- vs. higher-NLR groups: 30 [32.3\%] vs. 42 [54.5\%], $p=0.003$ ), and a higher likelihood of undergoing emergent surgery (lower- vs. higher-NLR groups: 5 [5.4\%] vs. $20[26.0 \%], p<0.001)$. 
Table 1

Patients' characteristics before and after propensity score matching

\begin{tabular}{|c|c|c|c|c|c|c|c|c|c|}
\hline & & \multicolumn{4}{|c|}{ Before matching } & \multicolumn{4}{|c|}{ After matching } \\
\hline & & $\begin{array}{l}\text { Total } \\
(n=170)\end{array}$ & $\begin{array}{l}\text { NLR } \leq \\
5 \\
(n=93)\end{array}$ & $\begin{array}{l}\text { NLR > } 5 \\
(n=77)\end{array}$ & $\begin{array}{l}\mathrm{P}- \\
\text { value }\end{array}$ & $\begin{array}{l}\text { Total } \\
(n=82)\end{array}$ & $\begin{array}{l}\text { NLR } \leq 5 \\
(n=41)\end{array}$ & $\begin{array}{l}\text { NLR > } 5 \\
(n=41)\end{array}$ & $\begin{array}{l}\mathrm{P} \text { - } \\
\text { value }\end{array}$ \\
\hline \multirow[t]{2}{*}{ Age } & $\leq 65 \mathrm{y} / \mathrm{o}$ & $\begin{array}{l}99 \\
(58.2 \%)\end{array}$ & $\begin{array}{l}56 \\
(60.2 \%)\end{array}$ & $\begin{array}{l}43 \\
(55.8 \%)\end{array}$ & 0.565 & $\begin{array}{l}49 \\
(59.8 \%)\end{array}$ & $\begin{array}{l}24 \\
(58.5 \%)\end{array}$ & $\begin{array}{l}25 \\
(61.0 \%)\end{array}$ & 0.822 \\
\hline & $>65 \mathrm{y} / \mathrm{o}$ & $\begin{array}{l}71 \\
(41.8 \%)\end{array}$ & $\begin{array}{l}37 \\
(39.8 \%)\end{array}$ & $\begin{array}{l}34 \\
(44.2 \%)\end{array}$ & & $\begin{array}{l}33 \\
(40.2 \%)\end{array}$ & $\begin{array}{l}17 \\
(41.5 \%)\end{array}$ & $\begin{array}{l}16 \\
(39.0 \%)\end{array}$ & \\
\hline \multirow[t]{2}{*}{ Sex } & Female & $\begin{array}{l}77 \\
(45.3 \%)\end{array}$ & $\begin{array}{l}47 \\
(50.5 \%)\end{array}$ & $\begin{array}{l}30 \\
(39.0 \%)\end{array}$ & 0.131 & $\begin{array}{l}35 \\
(42.7 \%)\end{array}$ & $\begin{array}{l}20 \\
(48.8 \%)\end{array}$ & $\begin{array}{l}15 \\
(36.6 \%)\end{array}$ & 0.264 \\
\hline & Male & $93(54.7 \%)$ & $\begin{array}{l}46 \\
(49.5 \%)\end{array}$ & $\begin{array}{l}47 \\
(61.0 \%)\end{array}$ & & $\begin{array}{l}47 \\
(57.3 \%)\end{array}$ & $\begin{array}{l}21 \\
(51.2 \%)\end{array}$ & $\begin{array}{l}26 \\
(63.4 \%)\end{array}$ & \\
\hline \multirow[t]{2}{*}{$\mathrm{Hb}$} & $\leq 10 \mathrm{~g} / \mathrm{dL}$ & $\begin{array}{l}75 \\
(44.1 \%)\end{array}$ & $\begin{array}{l}38 \\
(40.9 \%)\end{array}$ & $\begin{array}{l}37 \\
(48.1 \%)\end{array}$ & 0.347 & $\begin{array}{l}32 \\
(39.0 \%)\end{array}$ & $\begin{array}{l}16 \\
(39.0 \%)\end{array}$ & $\begin{array}{l}16 \\
(39.0 \%)\end{array}$ & 1.000 \\
\hline & $>10 \mathrm{~g} / \mathrm{dL}$ & $\begin{array}{l}95 \\
(55.9 \%)\end{array}$ & $\begin{array}{l}55 \\
(59.1 \%)\end{array}$ & $\begin{array}{l}40 \\
(51.9 \%)\end{array}$ & & $\begin{array}{l}50 \\
(61.0 \%)\end{array}$ & $\begin{array}{l}25 \\
(61.0 \%)\end{array}$ & $\begin{array}{l}25 \\
(61.0 \%)\end{array}$ & \\
\hline \multirow[t]{2}{*}{$\begin{array}{l}\text { WBC } \\
\text { count }\end{array}$} & $\leq 12000 / \mu \mathrm{L}$ & $\begin{array}{l}112 \\
(65.9 \%)\end{array}$ & $\begin{array}{l}81 \\
(87.1 \%)\end{array}$ & $\begin{array}{l}31 \\
(40.3 \%)\end{array}$ & $<.001$ & $\begin{array}{l}58 \\
(70.7 \%)\end{array}$ & $\begin{array}{l}29 \\
(70.7 \%)\end{array}$ & $\begin{array}{l}29 \\
(70.7 \%)\end{array}$ & 1.000 \\
\hline & $>12000 / \mu \mathrm{L}$ & $\begin{array}{l}58 \\
(34.1 \%)\end{array}$ & $\begin{array}{l}12 \\
(12.9 \%)\end{array}$ & $\begin{array}{l}46 \\
(59.7 \%)\end{array}$ & & $\begin{array}{l}24 \\
(29.3 \%)\end{array}$ & $\begin{array}{l}12 \\
(29.3 \%)\end{array}$ & $\begin{array}{l}12 \\
(29.3 \%)\end{array}$ & \\
\hline \multirow[t]{2}{*}{ CEA } & $\leq 5 \mathrm{ng} / \mathrm{mL}$ & $\begin{array}{l}70 \\
(41.2 \%)\end{array}$ & $\begin{array}{l}41 \\
(44.1 \%)\end{array}$ & $\begin{array}{l}29 \\
(37.7 \%)\end{array}$ & 0.397 & $\begin{array}{l}30 \\
(36.6 \%)\end{array}$ & $\begin{array}{l}15 \\
(40.5 \%)\end{array}$ & $\begin{array}{l}15 \\
(41.7 \%)\end{array}$ & 0.922 \\
\hline & $>5 \mathrm{ng} / \mathrm{mL}$ & $\begin{array}{l}100 \\
(58.8 \%)\end{array}$ & $\begin{array}{l}52 \\
(55.9 \%)\end{array}$ & $\begin{array}{l}48 \\
(62.3 \%)\end{array}$ & & $\begin{array}{l}43 \\
(52.4 \%)\end{array}$ & $\begin{array}{l}22 \\
(59.5 \%)\end{array}$ & $\begin{array}{l}21 \\
(58.3 \%)\end{array}$ & \\
\hline \multirow[t]{2}{*}{ Albumin } & $\leq 3.5 \mathrm{~g} / \mathrm{dL}$ & $\begin{array}{l}72 \\
(42.4 \%)\end{array}$ & $\begin{array}{l}30 \\
(32.3 \%)\end{array}$ & $\begin{array}{l}42 \\
(54.5 \%)\end{array}$ & 0.003 & $\begin{array}{l}38 \\
(46.3 \%)\end{array}$ & $\begin{array}{l}19 \\
(54.3 \%)\end{array}$ & $\begin{array}{l}19 \\
(54.3 \%)\end{array}$ & 1.000 \\
\hline & $>3.5 \mathrm{~g} / \mathrm{dL}$ & $\begin{array}{l}98 \\
(57.6 \%)\end{array}$ & $\begin{array}{l}63 \\
(67.7 \%)\end{array}$ & $\begin{array}{l}35 \\
(45.5 \%)\end{array}$ & & $\begin{array}{l}32 \\
(39.0 \%)\end{array}$ & $\begin{array}{l}16 \\
(45.7 \%)\end{array}$ & $\begin{array}{l}16 \\
(45.7 \%)\end{array}$ & \\
\hline DM & No & $\begin{array}{l}146 \\
(58.9 \%)\end{array}$ & $\begin{array}{l}82 \\
(88.2 \%)\end{array}$ & $\begin{array}{l}64 \\
(83.1 \%)\end{array}$ & 0.346 & $\begin{array}{l}65 \\
(79.3 \%)\end{array}$ & $\begin{array}{l}35 \\
(85.4 \%)\end{array}$ & $\begin{array}{l}30 \\
(73.2 \%)\end{array}$ & 0.173 \\
\hline & Yes & $\begin{array}{l}24 \\
(14.1 \%)\end{array}$ & $\begin{array}{l}11 \\
(11.8 \%)\end{array}$ & $\begin{array}{l}13 \\
(16.9 \%)\end{array}$ & & $\begin{array}{l}17 \\
(20.7 \%)\end{array}$ & $\begin{array}{l}6 \\
(14.6 \%)\end{array}$ & $\begin{array}{l}11 \\
(26.8 \%)\end{array}$ & \\
\hline Hepatitis & No & $\begin{array}{l}165 \\
(97.1 \%)\end{array}$ & $\begin{array}{l}90 \\
(96.8 \%)\end{array}$ & $\begin{array}{l}75 \\
(97.4 \%)\end{array}$ & 0.809 & $\begin{array}{l}79 \\
(96.3 \%)\end{array}$ & $\begin{array}{l}39 \\
(95.1 \%)\end{array}$ & $\begin{array}{l}40 \\
(97.6 \%)\end{array}$ & 0.556 \\
\hline & Yes & $5(2.9 \%)$ & $\begin{array}{l}3 \\
(3.2 \%)\end{array}$ & $\begin{array}{l}2 \\
(2.6 \%)\end{array}$ & & $\begin{array}{l}3 \\
(3.7 \%)\end{array}$ & $2(4.9 \%)$ & $\begin{array}{l}1 \\
(2.4 \%)\end{array}$ & \\
\hline $\begin{array}{l}\text { Liver } \\
\text { cirrhosis }\end{array}$ & No & $\begin{array}{l}168 \\
(98.8 \%)\end{array}$ & $\begin{array}{l}92 \\
(98.9 \%)\end{array}$ & $\begin{array}{l}76 \\
(98.7 \%)\end{array}$ & 0.893 & $\begin{array}{l}81 \\
(98.8 \%)\end{array}$ & $\begin{array}{l}41 \\
(100.0 \%)\end{array}$ & $\begin{array}{l}40 \\
(97.6 \%)\end{array}$ & 0.314 \\
\hline & Yes & $2(1.2 \%)$ & $\begin{array}{l}1 \\
(1.13 \%)\end{array}$ & $\begin{array}{l}1 \\
(1.3 \%)\end{array}$ & & $\begin{array}{l}1 \\
(1.2 \%)\end{array}$ & $\begin{array}{l}0 \\
(0.00 \%)\end{array}$ & $\begin{array}{l}1 \\
(1.4 \%)\end{array}$ & \\
\hline
\end{tabular}

NLR, neutrophil-to-platelet ratio; $y / o$, years old; $\mathrm{Hb}$, hemoglobin; WBC, white blood cell; CEA, carcinoembryonic antigen; DM, diabetes mellitus 


\begin{tabular}{|c|c|c|c|c|c|c|c|c|c|}
\hline \multirow[b]{2}{*}{ Location } & \multirow[b]{2}{*}{ Right } & \multicolumn{4}{|c|}{ Before matching } & \multicolumn{4}{|c|}{ After matching } \\
\hline & & $\begin{array}{l}63 \\
(37.1 \%)\end{array}$ & $\begin{array}{l}32 \\
(34.4 \%)\end{array}$ & $\begin{array}{l}31 \\
(40.3 \%)\end{array}$ & 0.432 & $\begin{array}{l}30 \\
(36.6 \%)\end{array}$ & $\begin{array}{l}13 \\
(31.7 \%)\end{array}$ & $\begin{array}{l}17 \\
(41.5 \%)\end{array}$ & 0.359 \\
\hline & Left & $\begin{array}{l}107 \\
(62.9 \%)\end{array}$ & $\begin{array}{l}61 \\
(65.6 \%)\end{array}$ & $\begin{array}{l}46 \\
(59.7 \%)\end{array}$ & & $\begin{array}{l}52 \\
(63.4 \%)\end{array}$ & $\begin{array}{l}28 \\
(68.3 \%)\end{array}$ & $\begin{array}{l}24 \\
(58.5 \%)\end{array}$ & \\
\hline \multirow[t]{2}{*}{$\begin{array}{l}\text { Emergent } \\
\text { surgery }\end{array}$} & No & $\begin{array}{l}142 \\
(85.3 \%)\end{array}$ & $\begin{array}{l}88 \\
(94.6 \%)\end{array}$ & $\begin{array}{l}57 \\
(74.0 \%)\end{array}$ & $<0.001$ & $\begin{array}{l}72 \\
(87.8 \%)\end{array}$ & $\begin{array}{l}36 \\
(87.8 \%)\end{array}$ & $\begin{array}{l}36 \\
(87.8 \%)\end{array}$ & 1.000 \\
\hline & Yes & $\begin{array}{l}25 \\
(14.7 \%)\end{array}$ & $\begin{array}{l}5 \\
(5.4 \%)\end{array}$ & $\begin{array}{l}20 \\
(26.0 \%)\end{array}$ & & $\begin{array}{l}10 \\
(12.2 \%)\end{array}$ & $\begin{array}{l}5 \\
(12.2 \%)\end{array}$ & $\begin{array}{l}5 \\
(12.2 \%)\end{array}$ & \\
\hline \multirow[t]{2}{*}{$\begin{array}{l}\text { Histology } \\
\text { type }\end{array}$} & Adenocarcinoma & $\begin{array}{l}146 \\
(85.9 \%)\end{array}$ & $\begin{array}{l}78 \\
(83.9 \%)\end{array}$ & $\begin{array}{l}68 \\
(88.3 \%)\end{array}$ & 0.408 & $\begin{array}{l}67 \\
(81.7 \%)\end{array}$ & $\begin{array}{l}34 \\
(82.9 \%)\end{array}$ & $\begin{array}{l}33 \\
(80.5 \%)\end{array}$ & 0.775 \\
\hline & $\begin{array}{l}\text { Mucinous } \\
\text { carcinoma }\end{array}$ & $\begin{array}{l}24 \\
(14.1 \%)\end{array}$ & $\begin{array}{l}15 \\
(16.1 \%)\end{array}$ & $\begin{array}{l}9 \\
(11.7 \%)\end{array}$ & & $\begin{array}{l}15 \\
(18.3 \%)\end{array}$ & $\begin{array}{l}7 \\
(17.1 \%)\end{array}$ & $\begin{array}{l}8 \\
(19.5 \%)\end{array}$ & \\
\hline Histology & $\begin{array}{l}\text { Well } \\
\text { differentiated }\end{array}$ & $11(6.5 \%)$ & $\begin{array}{l}9 \\
(9.7 \%)\end{array}$ & $\begin{array}{l}2 \\
(2.6 \%)\end{array}$ & 0.166 & $\begin{array}{l}3 \\
(3.7 \%)\end{array}$ & $1(2.4 \%)$ & $\begin{array}{l}2 \\
(4.9 \%)\end{array}$ & 0.446 \\
\hline \multirow[t]{2}{*}{ Grade } & Moderate & $\begin{array}{l}133 \\
(78.2 \%)\end{array}$ & $\begin{array}{l}71 \\
(76.3 \%)\end{array}$ & $\begin{array}{l}62 \\
(80.5 \%)\end{array}$ & & $\begin{array}{l}65 \\
(79.3 \%)\end{array}$ & $\begin{array}{l}31 \\
(75.6 \%)\end{array}$ & $\begin{array}{l}30 \\
(82.9 \%)\end{array}$ & \\
\hline & Poor & $\begin{array}{l}26 \\
(15.3 \%)\end{array}$ & $\begin{array}{l}13 \\
(14.0 \%)\end{array}$ & $\begin{array}{l}13 \\
(16.9 \%)\end{array}$ & & $\begin{array}{l}14 \\
(17.1 \%)\end{array}$ & $\begin{array}{l}9 \\
(22.0 \%)\end{array}$ & $\begin{array}{l}5 \\
(12.2 \%)\end{array}$ & \\
\hline \multirow[t]{3}{*}{ T stage } & 2 & $1(0.6 \%)$ & $0(0 \%)$ & $\begin{array}{l}1 \\
(1.3 \%)\end{array}$ & 0.338 & $\begin{array}{l}1 \\
(1.2 \%)\end{array}$ & $0(0 \%)$ & $\begin{array}{l}1 \\
(2.4 \%)\end{array}$ & 0.602 \\
\hline & 3 & $\begin{array}{l}24 \\
(14.1 \%)\end{array}$ & $\begin{array}{l}11 \\
(11.8 \%)\end{array}$ & $\begin{array}{l}13 \\
(16.9 \%)\end{array}$ & & $\begin{array}{l}12 \\
(14.6 \%)\end{array}$ & $\begin{array}{l}6 \\
(14.6 \%)\end{array}$ & $\begin{array}{l}6 \\
(14.6 \%)\end{array}$ & \\
\hline & 4 & $\begin{array}{l}145 \\
(85.3 \%)\end{array}$ & $\begin{array}{l}82 \\
(88.2 \%)\end{array}$ & $\begin{array}{l}63 \\
(81.8 \%)\end{array}$ & & $\begin{array}{l}69 \\
(84.1 \%)\end{array}$ & $\begin{array}{l}35 \\
(85.4 \%)\end{array}$ & $\begin{array}{l}34 \\
(82.9 \%)\end{array}$ & \\
\hline $\mathrm{N}$ stage & 0 & $\begin{array}{l}89 \\
(52.4 \%)\end{array}$ & $\begin{array}{l}49 \\
(52.7 \%)\end{array}$ & $\begin{array}{l}40 \\
(51.9 \%)\end{array}$ & 0.268 & $\begin{array}{l}45 \\
(54.9 \%)\end{array}$ & $\begin{array}{l}21 \\
(51.2 \%)\end{array}$ & $\begin{array}{l}24 \\
(58.5 \%)\end{array}$ & 0.174 \\
\hline & 1 & $\begin{array}{l}54 \\
(31.8 \%)\end{array}$ & $\begin{array}{l}33 \\
(35.5 \%)\end{array}$ & $\begin{array}{l}21 \\
(27.3 \%)\end{array}$ & & $\begin{array}{l}25 \\
(30.5 \%)\end{array}$ & $\begin{array}{l}16 \\
(39.0 \%)\end{array}$ & $\begin{array}{l}9 \\
(22.0 \%)\end{array}$ & \\
\hline & 2 & $\begin{array}{l}27 \\
(15.9 \%)\end{array}$ & $\begin{array}{l}11 \\
(11.9 \%)\end{array}$ & $\begin{array}{l}16 \\
(20.8 \%)\end{array}$ & & $\begin{array}{l}12 \\
(14.6 \%)\end{array}$ & $4(9.8 \%)$ & $\begin{array}{l}8 \\
(19.5 \%)\end{array}$ & \\
\hline
\end{tabular}

After one-to-one propensity score matching, no clinicopathological factor was significantly different between the higherand lower-NLR groups (Table 1). In patients with stage I to III perforated colon cancer, the 3- and 5-year overall survival rates were $75 \%$ and $61 \%$, respectively, in the lower-NLR group, whereas those of the higher-NLR group were $80 \%$ and $67 \%$, respectively. We also analyzed the 3-and 5-year overall survival in the subgroups of patients with stage II and III perforated colon cancer. We found that, in patients with stage II perforated colon cancer, the 3- and 5-year overall survival rates were $90 \%$ and $84 \%$ in the lower-NLR group, respectively, whereas those of the higher-NLR group were $87 \%$ and $74 \%$, respectively. Patients with stage III perforated colon cancer had 3- and 5-year overall survival rates of $60 \%$ and $41 \%$ in the lower-NLR group, respectively, whereas those of the higher-NLR group were $69 \%$ and $55 \%$, respectively. The difference in overall survival rates between the higher- and lower-NLR groups in each subgroup comparison was not significant ( $p=0.637$ for stages I-III, $p=0.512$ for stage II, and $p=0.500$ for stage III; Fig. 2). 
Disease-free survival was also not significantly different between the higher- and lower-NLR groups. In patients with stage I to III perforated colon cancer, the 3- and 5-year disease-free survival rates were both $70 \%$ in the lower-NLR group, whereas those of the higher-NLR group were $69 \%$ and $63 \%$, respectively. In patients with stage II cancer, the 3-and 5-year diseasefree survival rates were both $84 \%$ in the lower-NLR group, whereas those of the higher-NLR group were $74 \%$ and $69 \%$, respectively. In patients with stage III cancer, the 3- and 5-year disease-free survival rates were 55\% in both the lower- and higher-NLR group, whereas the corresponding values in the higher-NLR group were $58 \%$ and $50 \%$, respectively $(p=0.827$ for stage I-III, $p=0.296$ for stage II, and $p=0.785$ for stage III; Fig. 3).

\section{Discussion}

Chronic inflammation-induced carcinogenesis is a commonly accepted mechanism for several malignancies [3]. In colon cancer, systemic inflammation not only affects cytokines but also promotes angiogenesis and metastasis formation [12]. Therefore, several systemic inflammatory-associated markers, such as C-reactive protein, lymphocyte-to-monocyte ratio, platelet-to-lymphocyte ratio, and NLR, have been used as outcome predictors [4-7].

Tumor-associated cytokines and growth factors enter the circulation causing a systemic response. These factors, which cause tumor angiogenesis and possibly neoplasm progression, may lead to an increase in the neutrophil count [13]. Alternatively, the absolute lymphocyte count is assumed to reflect the immune response of patients with cancer; lymphopenia is also correlated with worse outcomes in patients with colorectal cancer [14]. Therefore, the NLR can be considered a parameter that reflects the relationship between pro-tumor inflammatory and anti-tumor immune factors.

Multiple studies have focused on the association between preoperative NLR and colon cancer outcomes. A normal NLR is considered to range between 0.78 and 3.53 [15]. A higher NLR $(>5)$ has been identified as a prognostic factor for poor overall survival and early recurrence $[9,10,16]$; however, in our study, patients with perforated colon cancer and higher NLR showed no significant differences in overall survival and disease-free survival (overall survival: $p=0.637$; disease-free survival: $p=0.827)$.

A higher NLR has also been associated with higher $\mathrm{T}$ and $\mathrm{N}$ stage and a higher incidence of extramural venous invasion [17]; however, in our study, T and N staging was not associated with a higher NLR. In patients with perforated colon cancer, regardless of whether they are showing clinical signs of peritonitis, perforation-related infection or inflammation is difficult to detect. Therefore, in these patients, the elevated neutrophil level associated with infection may be higher than the normal preoperative NLR. Therefore, pre-operative NLR may be unable to reflect the balance between cancer-related inflammation and the immune response in patients with perforated colon cancer.

This study had several limitations. First, this was a single-center retrospective study involving a small number of patients. Second, preoperative data were collected using the latest data point before surgery, either in the emergency room or during admission if elective surgery was performed. Third, the definition of perforation was based on pathological reports. Some patients who might have initially presented with local abscess formation and not peritoneal signs could have been enrolled in our study and undergone further elective surgery rather than emergent surgery. Therefore, the results may not reflect equivalent clinical conditions among patients. Further larger studies are required to validate our findings.

\section{Conclusion}

A higher NLR $(N L R>5)$ is a well-known risk factor for survival in resectable colon cancer. However, in patients with stage I to III perforated colon cancer, higher NLR did not correlate with poor outcomes, which limits its use in predicting survival outcomes.

\section{List Of Abbreviations}


NLR - Neutrophil-to-lymphocyte ratio; PSM - propensity score matching; OS - overall survival; DFS - disease-free survival; WBC - white blood cell

\section{Declarations}

Ethics approval and consent to participate: This study was reviewed and approved by Chang Gung Memorial Hospital's Institutional Review Board (IRB) (IRB approval number: 202000133B0). The IRB waived the requirement of obtaining informed consent from the participants.

Consent for publication: Not applicable.

Availability of data and materials: The authors confirm that all data are fully available without restrictions on reasonable request.

Competing interest: The authors declare that they have no competing interest.

Funding: Not applicable.

Authors' contributions: All authors contributed to the study conception and design. Material preparation, data collection, and analysis were performed by Chun-Yi Wu. The first draft of the manuscript was written by Chun-Yi Wu and was supervised by Jy-Ming Chiang. All authors commented on previous versions of the manuscript. All authors read and approved the final manuscript.

\section{Acknowledgments}

We would like to express our special thanks to our chair professor Jy-Ming Chiang for his advice and support.

\section{References}

1. Bray F, Ferlay J, Soerjomataram I, Sigel RL, Torre LA, Jemal A. Global cancer statistics 2018: GLOBOCAN estimates of incidence and mortality worldwide for 36 cancers in 185 countries. CA Cancer J Clin. 2018;68:394-424.

2. Colotta F, Allavena P, Sica A, Garlanda C, Mantovani A. Cancer-related inflammation, the seventh hallmark of cancer: links to genetic instability. Carcinogenesis. 2009;30:1073-81.

3. Coussens LM, Werb Z. Inflammation and cancer. Nature. 2002;420:860-7.

4. Absenger G, Szkandera J, Pichler M, Stotz M, Arminger F, Weissmueller M, et al. A derived neutrophil to lymphocyte ratio predicts clinical outcome in stage II and III colon cancer patients. Br J Cancer. 2013;109:395-400.

5. Bailon-Cuadrado M, Choolani-Bhojwani E, Tejero-Pintor FJ, Sanchez-Gonzalez J, Rodriguez-Lopez M, Perez-Saborido $B$, et al. Preoperative platelet-lymphocyte ratio is an independent factor of poor prognosis after curative surgery for colon cancer. Updates Surg. 2018;70:33-9.

6. Ding PR, An X, Zhang RX, Fang YJ, Li LR, Chen G, et al. Elevated preoperative neutrophil to lymphocyte ratio predicts risk of recurrence following curative resection for stage IIA colon cancer. Int J Colorectal Dis. 2010;25:1427-33.

7. Roxburgh CSD, McMillan DC. Role of systemic inflammatory response in predicting survival in patients with primary operable cancer. Future Oncol. 2010;6:149-63.

8. Chua W, Charles KA, Baracos VE, Clarke SJ. Neutrophil/lymphocyte ratio predicts chemotherapy outcomes in patients with advanced colorectal cancer. Br J Cancer. 2011;104:1288-95.

9. Tsai PL, Su WJ, Leung WH, Lai CT, Liu CK. Neutrophil-lymphocyte ratio and CEA level as prognostic and predictive factors in colorectal cancer: a systematic review and meta-analysis. J Cancer Res Ther. 2016;12:582-9. 
10. Walsh SR, Cook EJ, Goulder F, Justin TA, Keeling NJ. Neutrophil-lymphocyte ratio as a prognostic factor in colorectal cancer. J Surg Oncol. 2005;91:181-4.

11. Chiang SF, Hung HY, Tang R, Changchien CR, Chen JS, You YT, et al. Can neutrophil-to-lymphocyte ratio predict the survival of colorectal cancer patients who have received curative surgery electively? Int J Colorectal Dis.

2012;27:1347-57.

12. Terzic J, Grivennikov S, Karin E, Karin M. Inflammation and colon cancer. Gastroenterology. 2010;138:2101-14.e5.

13. Halazun KJ, Hardy MA, Rana AA, Woodland DC 4th, Luyten EJ, Mahadev S, et al. Negative impact of neutrophillymphocyte ratio on outcome after liver transplantation for hepatocellular carcinoma. Ann Surg. 2009;250:141-51.

14. Ceze N, Thibault G, Goujon G, Viguier J, Watier H, Dorval E, et al. Pre-treatment lymphopenia as a prognostic biomarker in colorectal cancer patients receiving chemotherapy. Cancer Chemother Pharmacol. 2011;68:1305-13.

15. Forget P, Khalifa C, Defour JP, Latinne D, Van Pel MC, De Kock M. What is the normal value of the neutrophil-tolymphocyte ratio? BMC Res Notes. 2017;10:12.

16. Mallappa S, Sinha A, Gupta S, Chadwick SJD. Preoperative neutrophil to lymphocyte ratio $>5$ is a prognostic factor for recurrent colorectal cancer. Colorectal Dis. 2013;15:323-8.

17. Pine JK, Morris E, Hutchins GG, West NP, Jayne DG, Quirke P, et al. Systemic neutrophil-to-lymphocyte ratio in colorectal cancer: the relationship to patient survival, tumour biology and local lymphocytic response to tumour. $\mathrm{Br} J$ Cancer. 2015;113:204-1

\section{Figures}

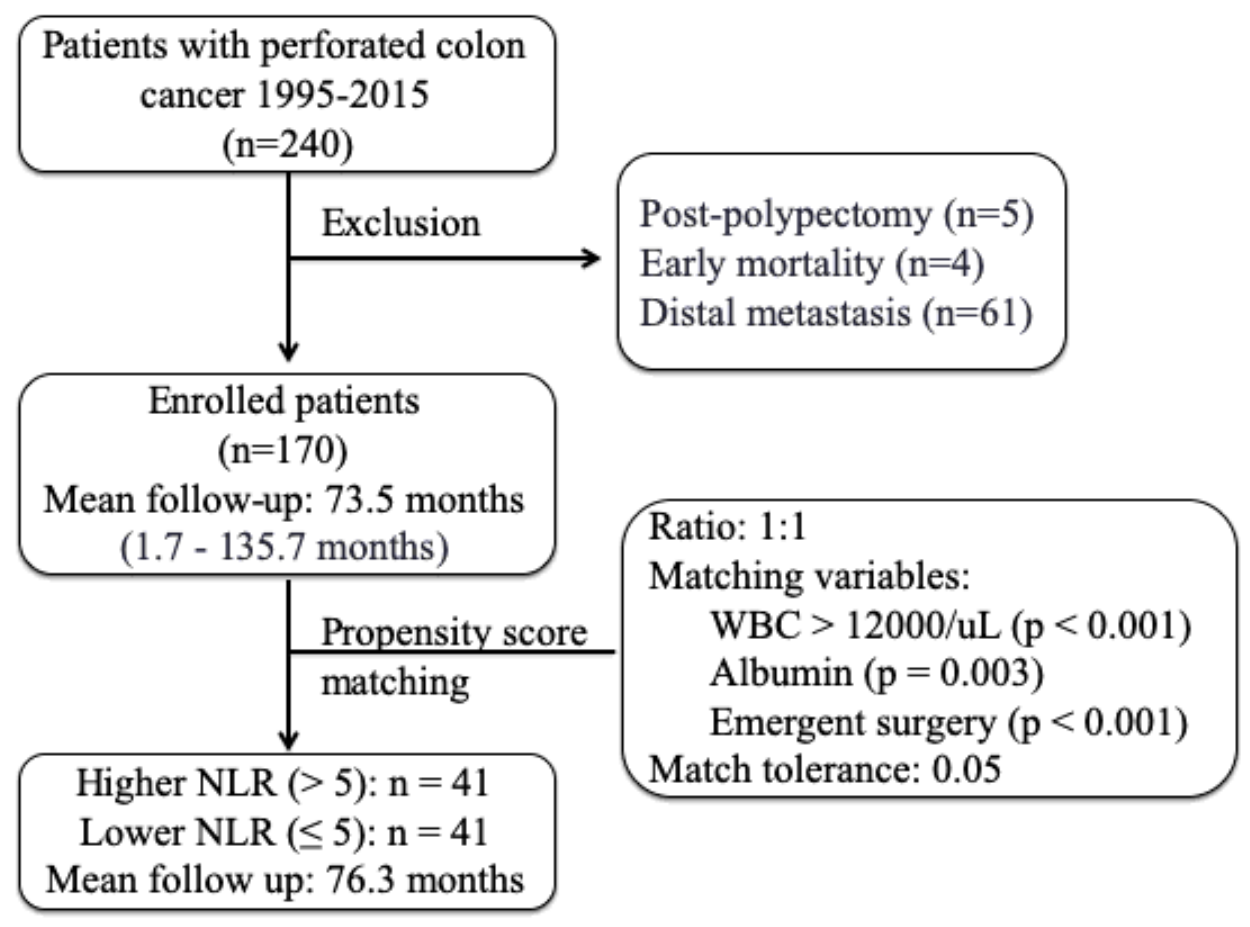

\section{Figure 1}

Flow chart of the study sample WBC, white blood cell; NLR, neutrophil-to-lymphocyte ratio 

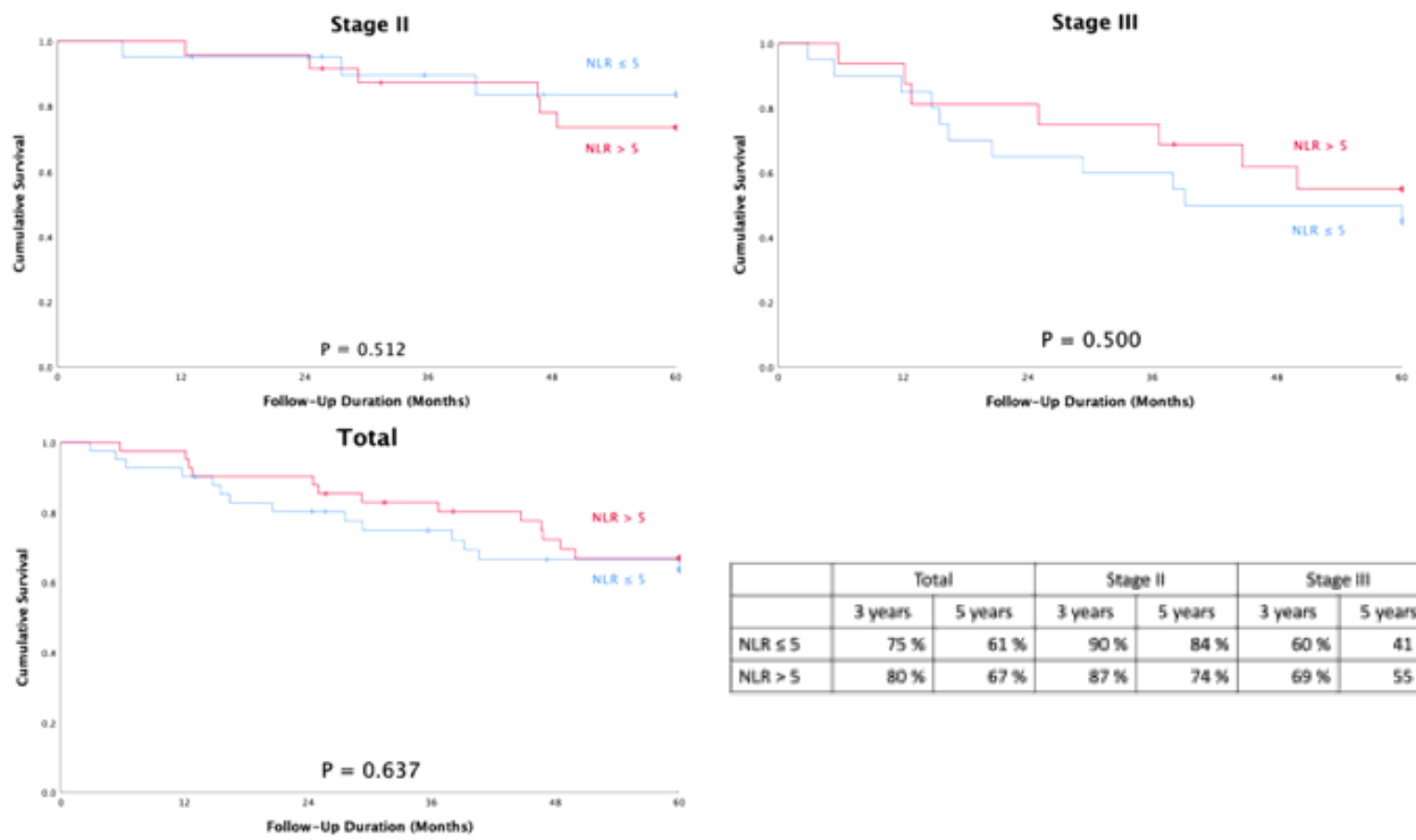

\begin{tabular}{|c|c|c|c|c|c|c|}
\hline & \multicolumn{2}{|c|}{ Total } & \multicolumn{2}{|c|}{ Sence II } & \multicolumn{2}{|c|}{ Stage ill } \\
\hline & 3 years & 5 years & 3 years & 5 years & 3 years & 5 years \\
\hline NLR $\leq 5$ & $75 x$ & $61 \%$ & $90 \%$ & $84 \times$ & $60 \%$ & $41 \%$ \\
\hline NLR $>5$ & $80 \%$ & $67 \%$ & $87 \%$ & $74 \%$ & $69 \%$ & $55 \times$ \\
\hline
\end{tabular}

Figure 2

Overall survival rates between patients with higher- and lower-NLR groups NLR, neutrophil-to-lymphocyte ratio
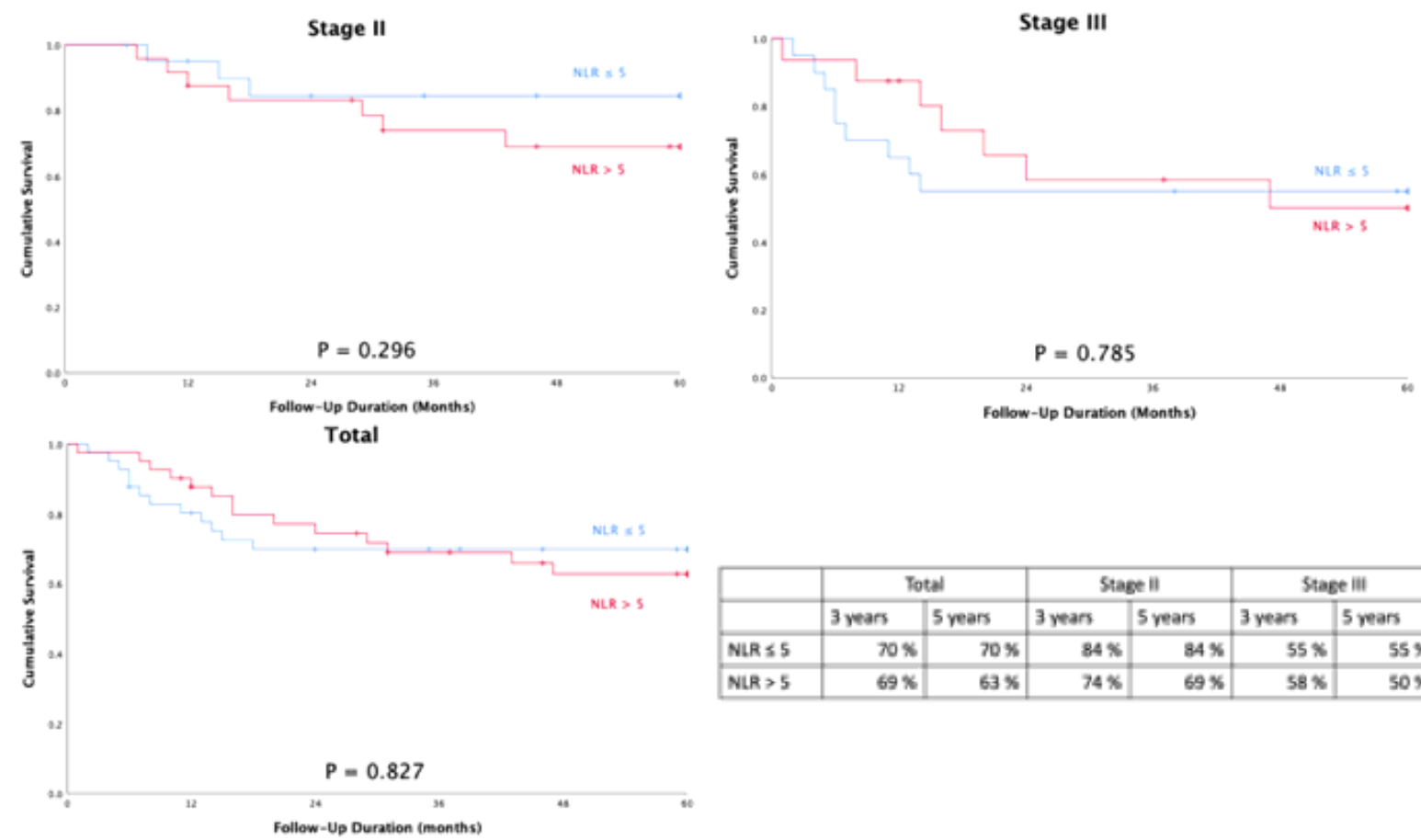

\begin{tabular}{|c|c|c|c|c|c|c|}
\hline & \multicolumn{2}{|c|}{ Total } & \multicolumn{2}{|c|}{ Stage il } & \multicolumn{2}{|c|}{ Stage ili } \\
\hline & 3 years & 5 years & 3 years & 5 years & 3 years & 5 years \\
\hline NLR $\leq 5$ & $70 \%$ & $70 \%$ & $84 \%$ & $84 \%$ & $55 \times$ & $55 \%$ \\
\hline NLR $>5$ & $69 x$ & $63 \%$ & $74 \%$ & $69 \times$ & $58 \%$ & $50 \%$ \\
\hline
\end{tabular}

Figure 3

Disease-free survival rates between patients with higher-and lower-NLR groups NLR, neutrophil-to-lymphocyte ratio 